\section{Conclusion}

So where do we stand today? We now appreciate that CX3CR1 and presumably fractalkine are important players in atherogenesis, and that CX3CR1 contains polymorphisms that modulate its adhesive and signaling functions and are associated with reduced risk of atherosclerotic cardiovascular disease. But to truly understand the mechanism of CX3CR1 and other genes in a highly complex and chronic disease process such as atherosclerosis, new practical intermediate markers are required in both experimental models and clinical studies.

1. Lesnik, P., Haskell, C.A., and Charo, I.F. 2003 Decreased atherosclerosis in $\mathrm{CX}_{3} C R 1^{-/-}$mice reveals a role for fractalkine in atherogenesis. J. Clin. Invest. 111:333-340. doi:10.1172/JCI200315555.

2. McDermott, D.H., et al. 2003. Chemokine receptor mutant CX3CR1-M280 has impaired adhesive function and correlates with protection from cardiovascular disease in man. J. Clin. Invest. 111:1241-1250. doi:10.1172/ JCI200316790.

3. Bazan, J.F., et al. 1997. A new class of membranebound chemokine with a CX3C motif. Nature. 385:640-644.

4. Pan, Y., et al. 1997. Neurotactin, a membraneanchored chemokine upregulated in brain inflammation. Nature. 387:611-617.

5. Imai, T., et al. 1997. Identification and molecular characterization of fractalkine receptor CX3CR1, which mediates both leukocyte migration and adhesion. Cell. 91:521-530.

6. Tsou, C.L., Haskell, C.A., and Charo, I.F. 2001. Tumor necrosis factor-alpha-converting enzyme mediates the inducible cleavage of fractalkine. J. Biol. Chem. 276:44622-44626.

7. Garton, K.J., et al. 2001. Tumor necrosis factoralpha-converting enzyme (ADAM17) mediates the cleavage and shedding of fractalkine
(CX3CL1). J. Biol. Chem. 276:37993-38001.

8. Faure, S., et al. 2000. Rapid progression to AIDS in HIV+ individuals with a structural variant of the chemokine receptor CX3CR1. Science 287:2274-2277.

9. McDermott, D.H., et al. 2001. Association between polymorphism in the chemokine receptor CX3CR1 and coronary vascular endothelial dysfunction and atherosclerosis. Circ. Res. 89:401-407.

10. Moatti, D., et al. 2001. Polymorphism in the fractalkine receptor CX3CR1 as a genetic risk factor for coronary artery disease. Blood. 97:1925-1928.

11. Combadiere, C., et al. 2003. Decreased atherosclerotic lesion formation in CX3CR1/apolipoprotein E double knockout mice. Circulation. 107:1009-1016.

12. Hegele, R.A. 2002. SNP judgments and freedom of association. Arterioscler. Thromb. Vasc. Biol. 22:1058-1061

13. DeVries, M.E., et al. 2003. Genomic organization and evolution of the CX3CR1/CCR8 chemokine receptor locus. J. Biol. Chem. 278:11985-11994. doi:10.1074/jcb.M211422200.

\section{RANK ligand and the regulation of skeletal remodeling}

\author{
Norman H. Bell \\ Department of Medicine, Medical University of South Carolina, Charleston, \\ South Carolina, USA
}

J. Clin. Invest. 111:1120-1122 (2003). doi:10.1172/JCI200318358.

It has long been known that estrogen deficiency in animal models and postmenopausal women is associated with increased osteoclastic bone resorption and bone loss (1-5). In the past decade, several important discoveries of some of the key factors involved in osteoclast formation, survival, function, and regulation by estrogen have been made.

A number of hormones and cytokines modulate osteoclastogenesis by enhancing osteoclast differentiation, activation,

Address correspondence to: Norman H. Bell, Department of Medicine, Medical University of South Carolina, 114 Doughty Street, Charleston, South Carolina 29425, USA. Phone: (843) 876-5162; Fax: (843) 876-5163; E-mail: belln@musc.edu.

Conflict of interest: The author has declared that no conflict of interest exists.

Nonstandard abbreviations used: parathyroid hormone (PTH); receptor activator of NF- $\mathrm{KB}$ (RANK); RANK ligand (RANKL); osteoprotegerin (OPG); selective estrogen receptor modulator (SERM). lifespan, and function. These include parathyroid hormone (PTH), calcitriol, PTH-related protein, prostaglandin $\mathrm{E}_{2}$, thyroxine, and IL-11 (3-5). The formation of active osteoclasts requires M-CSF (1,3-6) and involves cell-to-cell contact between precursors of the monocyte/macrophage lineage and osteoblasts, marrow stromal cells, and $\mathrm{T}$ and $B$ cells. These cells express the receptor activator of NF- $\kappa B$ ligand (RANKL), a member of the TNF ligand family, which is essential for this process. RANKL attaches to RANK, a receptor on the cell surface of osteoclasts and osteoclast precursors, to stimulate proliferation and differentiation of cells to form the osteoclast phenotype and inhibit apoptosis. Osteoprotegerin (OPG), a soluble decoy receptor produced by osteoblasts, marrow stromal cells, and other cells, profoundly modifies the effects of RANKL by inhibiting RANKL/ RANK interaction (1, 3-5).
In laboratory animals, estrogen deficiency leads to increased osteoclastogenesis and bone loss, and inhibition of osteoclastogenesis is the major means by which estrogen prevents the loss of bone. This is accomplished by diminishing production of IL-1, IL-6, and TNF- $\alpha-$ cytokines that enhance production of M-CSF and RANKL (1, 3-5) - and downregulating NF-KB and RANKL-induced activation of JNK1 and osteoclastogenic activator protein-1 transcription factors c-Fos and c-Jun (7). Estrogen upregulates OPG (8) and TGF- $\beta$ (9), and TGF- $\beta$ increases OPG expression by osteoblasts and stromal cells (10) and inhibits bone resorption by increasing apoptosis of osteoclasts (11) (Figure 1).

\section{RANKL and postmenopausal bone loss}

Whether the OPG/RANKL/RANK system is involved in bone loss caused by estrogen deficiency in humans was not known until now. In this issue of the JCI (12), Eghbali-Fatourechi and colleagues employ an elegant set of experiments to examine the possible role of RANKL in postmenopausal bone loss. The authors obtained bone marrow mononuclear cells and used surface markers and flow cytometry to isolate and identify preosteoblastic marrow stromal cells, $\mathrm{T}$ lymphocytes, and B lymphocytes in groups of premenopausal women, untreated postmenopausal women, and 


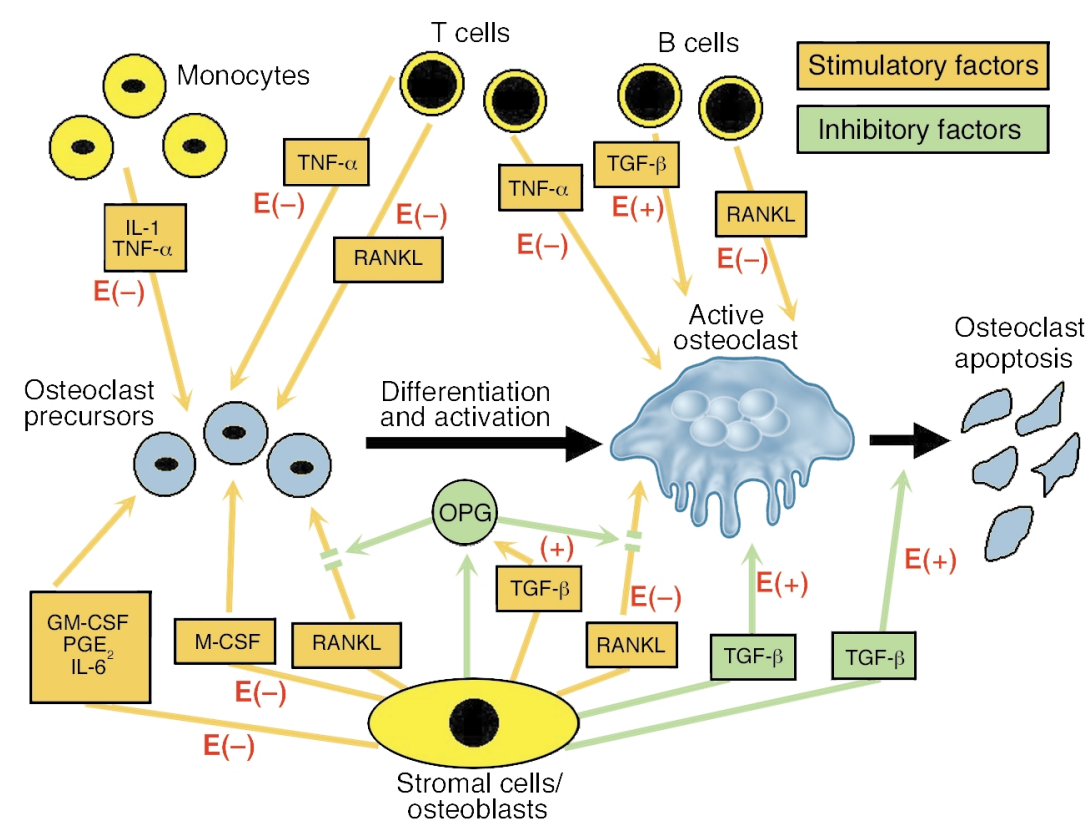

Figure 1

Regulation of osteoclast formation, function, and apoptosis by cytokines produced by bone marrow stromal cells, osteoblasts, monocytes, T cells, and B cells. Stimulatory factors are shown in orange and inhibitory factors in green. The effects of $E$ to enhance $\left(^{+}\right)$and inhibit $(-)$ the factors are shown in red.

postmenopausal women treated with estrogen. Characteristically, serum $17 \beta$-estradiol was reduced, and serum and urinary markers of bone resorption were increased in postmenopausal compared to premenopausal women and postmenopausal women treated with estrogen. Concentrations of serum OPG and RANKL were not different in the three groups. It was found that the levels of RANKL per cell, preosteoblasts, $\mathrm{T}$ cells, and B cells were increased by twoto three-fold in the untreated postmenopausal women and correlated positively with serum and urinary markers of bone resorption and negatively with serum $17 \beta$-estradiol in the three groups. As discussed in this article, the fact that serum RANKL was not different in the three groups indicates the necessity for investigating its concentration in the microenvironment of marrow. These results are important because they provide strong evidence that: (i) it is the upregulation of RANKL on bone marrow cells as opposed to increases in the number of $\mathrm{T}$ or B cells (as occurs in rodents) that plays a pathogenetic role in increased postmenopausal skeletal remodeling; (ii) the immune system is intimately involved in this process; and (iii) estrogen directly or indirectly modifies this process. The study also provides a rationale for the use of drugs that modify osteoclastogenesis in the treatment of estrogen deficiency-related bone loss.

\section{Effects of OPG and RANK gene mutations}

The importance of the OPG/RANKL/ RANK system in regulating osteoclastogenesis is underscored by the findings that OPG-deficient mice develop profound osteoclastogenesis and osteoporosis with fractures (13), and that mutations in OPG and RANK in humans cause unrestrained bone resorption and generalized bone disease. Inactivating mutations in TNFRSF11B, the TNF receptor superfamily member $11 \mathrm{~b}$ gene encoding OPG, causes a high turnover bone disorder variously called hereditary hyperphosphatasia, hyperostosis corticalis deformans juvenilis, craniotubular dysostosis with hyperphosphatasia, or juvenile Paget's disease $(14,15)$. The disorder is characterized by increased susceptibility to fractures, marked increases in skeletal remodeling with widened diaphyses and progressive deformities of long bones, deformities of the pelvis and vertebrae, and massive thickening of the calvarium. Histologically, the number of osteoclasts and osteoblasts is greatly increased. Activating mutations in TNFRSF11A, the TNF receptor superfamily member 11 a gene encoding RANK, causes two bone diseases. These are familial expansile osteolysis, which is characterized by focal expansile osteolytic bone lesions and generalized osteopenia (16), and expansile skeletal hyperphosphatasia, which is characterized by deafness, premature loss of teeth, progressive hyperostotic widening of long bones, enhanced bone remodeling, and intermittent hypercalcemia (17). Since OPG has been shown to reverse the bone disease in OPG-deficient mice (18), OPG should be a madeto-order means of therapy for these clinical disorders.

\section{Treatment of postmenopausal osteoporosis}

To date, drugs and hormones that act by inhibiting osteoclast-mediated bone resorption have been the mainstay of osteoporosis treatment and prevention of fractures. In addition to estrogen, these include bisphophonates, selective estrogen receptor modulators (SERMs), and calcitonin (19). Although estrogen reduces the incidence of fractures and colorectal cancer, it increases the incidence of coronary artery disease, stroke, breast cancer, and thromboembolic events (20). An increased incidence of uterine cancer is prevented by coadministration of progestins. Raloxifene, a SERM which prevents bone loss and fractures in postmenopausal women, inhibits the growth of uterine tissue and reduces the incidence of breast cancer but increases the incidence of thromboembolic phenomena $(19,21)$. Calcitonin inhibits osteoclastic bone resorption, an effect mediated by calcitonin receptors. However, calcitonin downregulates calcitonin receptors, and this may reduce its effectiveness (19). Bisphosphonates act by inactivating osteoclasts to increase bone mineral density and prevent fractures whereas longterm treatment with bisphosphonates produces microdamage accumulation and increased susceptibility to fractures in dogs (22). This potential complication of bisphosphonate therapy has not been reported in patients with osteoporosis but could occur as a consequence of inhibition of bone formation rate and remains a concern (21). 
New drugs that are under investigation to treat bone-resorbtion diseases include inhibitors of $\alpha_{v} \beta_{3}$ integrin, an adhesion receptor that mediates attachment of osteoclasts to bone surface $(5,19)$, and OPG. Indeed, in doseresponse studies lasting for two to three months, single doses of OPG, which inhibits both differentiation and activation of osteoclasts, were shown to profoundly inhibit bone resorption in postmenopausal women (23) and in patients with multiple myeloma or skeletal metastases caused by breast cancer (24).

Despite the breadth and depth of these seminal discoveries, there is still much more to be learned about basic bone biology and the mechanisms by which estrogen modulates bone metabolism.

1. Riggs, B.L., Khosla, S., and Melton, L.J. 2002 Sex steroids and the construction and conservation of the adult skeleton. Endocr. Rev. 23:279-302.

2. Pacifici, R. 1996. Estrogen, cytokines, and pathogenesis of postmenopausal osteoporosis. J. Bone Miner. Res. 11:1043-1051.

3. Manolagas, S.C. 2000. Birth and death of bone cells: basic regulatory mechanisms and implications for the pathogenesis and treatment of osteoporosis. Endocr. Rev. 21:115-137.

4. Suda, T., et al. 1999. Modulation of osteoclast differentiation and function by the new members of the tumor necrosis factor receptor and ligand families. Endocr. Rev. 20:345-357.

5. Duong, L.T., and Rodan, G.A. 2001. Regulation of osteoclast formation and function. Rev. Endocr. Metab. Disord. 2:95-104.

6. Tanaka, S., et al. 1993. Macrophage colony-stimulating factor is indispensable for both proliferation and differentiation of osteoclast progenitors. J. Clin. Invest. 91:257-263.

7. Strivastava, S., et al. 2001. Estrogen decreases osteoclast formation by down-regulating receptor activator of NF- $\mathrm{KB}$ ligand (RANKL)-induced JNK activation. J. Biol. Chem. 276:8836-8840.

8. Hofbauer, L.C., et al. 2000. The roles of osteoprotegerin and osteoprotegerin ligand in the paracrine regulation of bone resorption. J. Bone Miner. Res. 15:2-12.

9. Finkelman, R.D., Bell, N.H., Strong, D.D. Demers, L.M., and Baylink, D.J. 1992. Ovariectomy selectively reduces the concentration of transforming growth factor beta in rat bone: implications for estrogen deficiency-associated bone loss. Proc. Natl. Acad. Sci. U. S. A. 89:12190-12193.

10. Thirunavukkarasu, K.T., et al. 2001. Stimulation of osteoprotegerin (OPG) gene expression by transforming growth factor- $\beta$ (TGF- $\beta$ ). J. Biol. Chem. 276:36241-36250.

11. Weitzmann, M.N., et al. 2000. B lymphocytes inhibit human osteoclastogenesis by secretion of TGF 3 . J. Cell. Biochem. 78:318-324.

12. Eghbali-Fatourechi, G., et al. 2003. Role of RANK ligand in mediating increased bone resorption in early postmenopausal women. J. Clin. Invest. 111:1221-1230. doi:10.1172/JCI200317215.

13. Bucay, N., et al. 1998. Osteoprotegerin-deficient mice develop early onset osteoporosis and arterial calcification. Genes Dev. 12:1260-1268.

14. Whyte, M.P., et al. 2002. Osteoprotegerin deficiency and juvenile Paget's disease. N. Engl. J. Med. 347:175-184.

15. Cundy, T., et al. 2002. A mutation in the gene
TNFRSF11B encoding osteoprotegerin causes an idiopathic hyperphosphatasia phenotype. Hum Mol. Genet. 11:2119-2127.

16. Johnson-Pais, T.L., et al. 2002. Identification of a novel tandem duplication in exon I of the TNFRSF11A gene in two unrelated patients with familial expansile osteolysis. J. Bone Miner. Res. 18:376-380.

17. Whyte, M.P., and Hughes, A.E. 2002. Expansile skeletal hyperphosphatasia is caused by a 15 -base pair tandem duplication in TNFRSF11A encoding RANK and is allelic to familial expansile osteolysis. J. Bone Miner. Res. 18:376-380.

18. Min, H., et al. 2000. Osteoprotegerin reverses osteoporosis by inhibiting endosteal osteoclasts and prevents vascular calcification by blocking a process resembling osteoclastogenesis. J. Exp. Med. 192:463-474.

19. Rodan, G.A., and Martin, T.J. 2000. Therapeutic approaches to bone diseases. Science. 289:1508-1514.

20. Nelson, H.D., Humphrey, L.L., Nygren, P., Teutsch S.M., and Allan, J.D. 2002. Post-menopausal hormone replacement therapy: scientific review. JAMA. 288:872-881.

21. Ott, S.M., Oleksik, A., Lu, Y., Harper, K., and Lips, P. 2002. Bone histomorphometric and biochemical markers: results of a 2 -year placebo-controlled trial of raloxifene in post-menopausal women. J. Bone Miner. Res. 17:341-348.

22. Mashiba, T., et al. 2001. Effect of suppressed bone turnover by bisphosphonate on microdamage and biomechanical properties in clinically relevant skeletal sites in beagles. Bone. 28:524-531.

23. Bekker, P.J., et al. 2001. The effect of a single dose of osteoprotegerin in postmenopausal women. J. Bone Miner. Res. 16:348-360.

24. Body, J.J., et al. 2003. A Phase I study of AMGN 0007, a recombinant osteoprotegerin construct in patients with multiple myeloma or breast carcinoma related bone metastases. Cancer. 97(Suppl. 3):887-892.

\title{
Azathioprine: old drug, new actions
}

\author{
Jonathan S. Maltzman ${ }^{1,2}$ and Gary A. Koretzky ${ }^{1,3}$ \\ ${ }^{1}$ Signal Transduction Program, Abramson Family Cancer Research Institute, \\ ${ }^{2}$ Renal-Electrolyte and Hypertension Division, Department of Medicine, and \\ ${ }^{3}$ Department of Pathology and Laboratory Medicine, University of Pennsylvania School of \\ Medicine, Philadelphia, Pennsylvania, USA
}

J. Clin. Invest. 111:1122-1124 (2003). doi:10.1172/JCI200318384.

\section{Address correspondence to: Gary A.} Koretzky, Abramson Family Cancer Research Institute, University of Pennsylvania School of Medicine, 415 Biomedical Research Building II/III, 421 Curie Boulevard, Philadelphia, Pennsylvania 19104, USA. Phone: (215) 746-5522; Fax: (215) 746-5525; Email: Koretzky@mail.med.upenn.edu.

Conflict of interest: The authors have declared that no conflict of interest exists.

Nonstandard abbreviations used: 6-mercaptopurine (6-MP); 6-thioguanine (6-TG); T cell receptor (TCR); nuclear factor of activated T cells (NFAT); 6-thioguanine triphosphate (6-ThioGTP).
A knowledge of the biochemical loci of action of 6-MP in the inbibition of nucleic acid synthesis is not sufficient to explain the effects of the thiopurines on the immune system.

-Gertrude B. Elion (Winner of 1988 Nobel Prize in Medicine for "important principals of drug development"; codiscovered 6-MP and azathioprine with George Hitchings) (1)
Azathioprine is among the oldest pharmacologic immunosuppressive agents in use today. Initially developed as a long-lived prodrug of 6-mercaptopurine (6-MP), it was quickly found to have a more favorable therapeutic index. It was soon found that 6-MP could produce remissions in childhood acute leukemia (1), and later, that azathioprine could prolong renal allograft survival (2). Over the past 50 years, azathioprine has been used in the treatment of hematologic malignancies, rheumatologic diseases, solid organ transplantation, and inflammatory bowel disease.

The drug is a purine analog, and the accepted mechanism of action is at the level of DNA $(1,3)$. Both in vitro and in vivo, azathioprine is metabolized to 6-MP through reduction by glutathione and other sulphydryl-containing compounds and then enzymatically converted into 6-thiouric acid, 6-methyl-MP, and 6-thioguanine 